Page 1 of 4

\title{
Origin of the pseudogap phase: Precursor superconductivity versus a competing energy gap scenario
}

\author{
K. Levin ${ }^{1}$, Qijin Chen ${ }^{2}$, Ioan Kosztin ${ }^{3}$, Boldizsár Jankó ${ }^{4}$, Ying-Jer Kao ${ }^{1}$, and Andrew Iyengar ${ }^{1}$ \\ ${ }^{1}$ James Franck Institute, University of Chicago, 5640 South Ellis Avenue, Chicago, Illinois 60637, USA \\ ${ }^{2}$ National High Magnetic Field Lab, Tallahassee, Florida 32310, USA \\ ${ }^{3}$ Beckman Institute and Department of Physics, University of Illinois, Urbana, Illinois 61801, USA \\ ${ }^{4}$ Department of Physics, University of Notre Dame, Notre Dame, Indiana 46556, USA
}

\begin{abstract}
In the last few years evidence has been accumulating that there are a multiplicity of energy scales which characterize superconductivity in the underdoped cuprates. In contrast to the situation in BCS superconductors, the phase coherence temperature $T_{c}$ is different from the energy gap onset temperature $T^{*}$. In addition, thermodynamic and tunneling spectroscopies have led to the inference that the order parameter $\Delta_{s c}$ is to be distinguished from the excitation gap $\Delta$; in this way, pseudogap effects persist below $T_{c}$. It has been argued by many in the community that the presence of these distinct energy scales demonstrates that the pseudogap is unrelated to superconductivity. In this paper we show that this inference is incorrect. We demonstrate that the difference between the order parameter and excitation gap and the contrasting dependences of $T^{*}$ and $T_{c}$ on hole concentration $x$ and magnetic field $H$ follow from a natural generalization of BCS theory. This simple generalized form is based on a BCS-like ground state, but with self consistently determined chemical potential in the presence of arbitrary attractive coupling $g$. We have applied this mean field theory with some success to tunneling, transport, thermodynamics and magnetic field effects. We contrast the present approach with the phase fluctuation scenario and discuss key features which might distinguish our precursor superconductivity picture from that involving a competing order parameter.

cond-mat/0107275
\end{abstract}

One of the biggest questions which faces the high temperature superconductivity community is determining the origin of the pseudogap phase. It is now becoming clear that pseudogap effects are not restricted to the normal state alone. Moreover, they appear to persist over a wide range of the phase diagram, up to and possibly above optimal doping[1]]. Understanding the pseudogap phase is essential in order to find a proper replacement for BCS theory. Indeed, the failure of BCS theory is demonstrated most clearly in thermodynamical[2] and tunneling[3] data which have made it clear that the underlying normal phase below $T_{c}$ contains a (pseudo)gap in the excitation spectrum. The phase diagram, itself, indicates that the larger the pseudogap the lower is $T_{c}$ and in this way the pseudogap appears to compete with superconductivity. This competition has led many researchers to argue that $T_{c}$ and $T^{*}$ must necessarily originate from different physical mechanisms. A recent D- density wave (DDW) theory [4 presents a concrete realization of the "competing energy gap" scenario, first conjectured by Loram and co-workers[2].

The present paper summarizes work from our group [5 10] which is based on a precursor superconductivity approach to understanding the pseudogap phase. We have been arguing for some time now that pseudogap effects necessarily persist below $T_{c}$, and moreover, appear to compete with superconductivity - despite their common origin. Our approach is based on a simple physical picture [1]] which interpolates smoothly between BCS theory and Bose Einstein condensation, and on its realization as first studied by Leggett[12], Nozieres [13] and Randeria[14] and their respective co-workers. Our main contribution has been to extend the Leggett ground state picture to non zero temperatures in a self consistent fashion, so as to incorporate pseudogap effects which were absent in earlier finite temperature calculations. This is a manifestly mean field approach which should be contrasted with the phase fluctuation scenario[15]. The latter builds on strict BCS theory (which is a special case of our more general theory), but goes beyond to include fluctuations of the order parameter phase, which we neglect here. It should also be stressed that a strong case can be made for focusing as we do here on an alternative mean field theory, as distinguished from a 
fluctuation scheme. A nice summary of the experimental support for this viewpoint, (principally based on the observed narrow fluctuation regime), can be found in Ref. [16].

The key assumption underlying our theoretical approach is that the ground state is of the generalized BCS form with wave-function

$\Psi_{0}=\prod_{\mathbf{k}}\left[u_{\mathbf{k}}+v_{\mathbf{k}} c_{\mathbf{k}}^{\dagger} c_{-\mathbf{k}}^{\dagger}\right]|0\rangle$

Here, however, the chemical potential $\mu$ is determined self consistently with variable attractive coupling constant $g$. As $g$ increases, $\mu$ progressively decreases, ultimately becoming negative in the "bosonic" regime. In this approach it should be clear that superconductivity need not be associated with an underlying Fermi liquid state. Without doing any calculations one can make a number of inferences about the finite temperature behavior associated with $\mathrm{Eq}(1)$. It is clear that at sufficiently strong coupling, the onset for pair formation $T^{*}$ will be different from $T_{c}[13]$. It then follows[6] that the excitation gap $\Delta$ and mean field order parameter $\Delta_{s c}$ are necessarily distinct- except, however, at zero temperature, where there is full condensation of all pairs ( $\Delta=\Delta_{s c}$ ), as is implicit in Eq. (1).

It should not be surprising, given the BCS-like form of the ground state wave-function, that one arrives at self consistent equations for $\Delta$ and the chemical potential $\mu$ which are essentially those of BCS theory. Here, however, the dispersion relation for fermionic excitations is

$E_{\mathbf{k}}=\sqrt{\Delta^{2} \varphi_{\mathbf{k}}^{2}+\left(\xi_{\mathbf{k}}-\mu\right)^{2}}, \quad \Delta^{2}=\Delta_{s c}^{2}+\Delta_{p g}^{2}$

where $\varphi_{\mathbf{k}}$ represents $s$ - or $d$-wave symmetry and the essential deviation from BCS theory derives from the presence of the pseudogap $\Delta_{p g}(T)$ which must be determined from a third self consistency condition (not given here). This latter equation, in effect, differentiates our approach from others [2, 4 ] which also have a BCS-like structure, but in which $\Delta_{p g}$ is presumed to arise from a non-pairing channel. Here, by contrast, the pseudogap arises from the stronger (than BCS) attractive interaction, and is associated with excitations of finite momentum pairs. The underlying microscopic theory behind our approach is based on a T-matrix decoupling scheme of Kadanoff and Martin[17] which is discussed elsewhere[9]. This T-matrix can be thought of as a propagator for pairs which, in turn, depends on the single particle self energy. The latter, in turn, depends on the T-matrixso that particles and pairs interact and no higher order correlation functions are included. Stated simply, whereas BCS is a mean field treatment of the fermions (and condensate), here we go beyond BCS to include non-condensed (and essentially non-interacting) pairs which are correspondingly treated at the mean field level.

With this well defined many body scheme, one can compute two particle properties such as as the ac conductivity and superfluid density $\rho_{s}[$ [7]. The conductivity diagrams are related to the well known Maki-Thompson and Aslamazov-Larkin terms (but with additional self energy insertions[18]). A relatively simple expression for $\rho_{s}$ results, which is of the BCS form, but with a prefactor of $\Delta_{s c}^{2}(T)$, where everywhere else in the expression, the full energy gap $\Delta$ appears. What needs to be stressed here is that $\rho_{s}$ reaches zero (at $T_{c}$ ) "prematurely" before the fermionic excitation gap $\Delta$ vanishes and that this occurs because there are bosonic degrees of freedom (finite momentum pair excitations) in addition to the usual $d$-wave fermions. The presence of additional bosonic excitations is, perhaps, the simplest way to understand the experimental observation that $\rho_{s}$ scales with $T / T_{c}(x)$ rather than with $T / \Delta(x)$.

In order to pass from coupling constant $g$ to hole concentration $x$, we note that the effective coupling (which always enters in a dimensionless form as a ratio to the bandwidth) is increased with underdoping, since electronic energy scales decrease as the Mott insulator is approached. Assuming $g$ is $x$ independent, and with one free parameter[7], we find a reasonable fit to the phase diagram as shown in Fig. 1. Indeed, the measured "universality" in a plot of $\rho_{s}(T, x) / \rho_{s}(0, x)$ vs $T / T_{c}(x)$ appears to be rather well satisfied within our theory, as is shown in Fig. 2 below- along with a comparison of data from Ref.[19]. It should be stressed that this methodology for incorporating Mott insulating physics can be viewed as a device for fitting the phase diagram and is not an essential component of the good agreement between theory and experiment shown in Fig. 2.

The bosons in the present theory, have a quasi-ideal gas character, as might be expected based on the fully condensed ground state wavefunction, and this leads naturally to a low temperature rounding $\left(T^{3 / 2}\right.$, which 


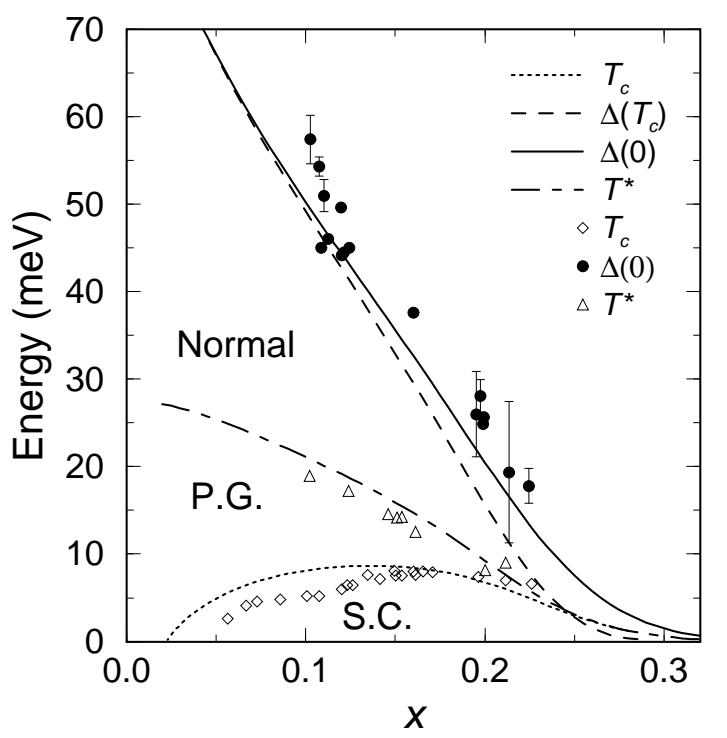

Figure 1. Comparison between theoretically computed (with one fitting parameter) and measured phase diagram.

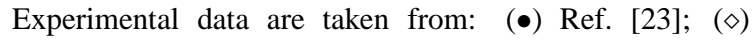
Ref. [24]; $(\triangle)$ Ref. [25]. For details see Ref. [7].

enters with $\Delta_{s c}^{2}$ ) of the inverse square of the penetration depth. Indeed, it is difficult to find samples where there is no low $T$ deviation from the expected $d$-wave linear dependence, [20] although this rounding has generally been attributed to impurity effects (and fitted to $T^{2}$ ). It should be stressed that the bosonic degrees of freedom will show up quite generally in the finite frequency conductivity as well.

The presence of a pseudogap in the fermionic spectrum at $T_{c}$ has a number of important consequences. Because of the BCS like structure of the equation for $\Delta$, it follows that $T_{c}$ will be suppressed as $\Delta_{p g}$ increases. Physically, this is a consequence of the depressed density of states which makes it difficult for fermions to pair. This, in turn, is consistent with the general trends in the phase diagram with increased underdoping. The presence of this gap is also responsible for the stronger sensitivity to pair breaking perturbations, i.e. magnetic fields [21] and impurity substitutions, seen in $T_{c}$, but not in $T^{*}$. This latter observation should counter the wide-spread inference that the contrasting behavior of $T_{c}$ and $T^{*}$ with respect to $H$ and other perturbations is suggestive of a competing rather than precursor origin of the pseudogap. Finally, the presence of a pseudogap at $T_{c}$ raises

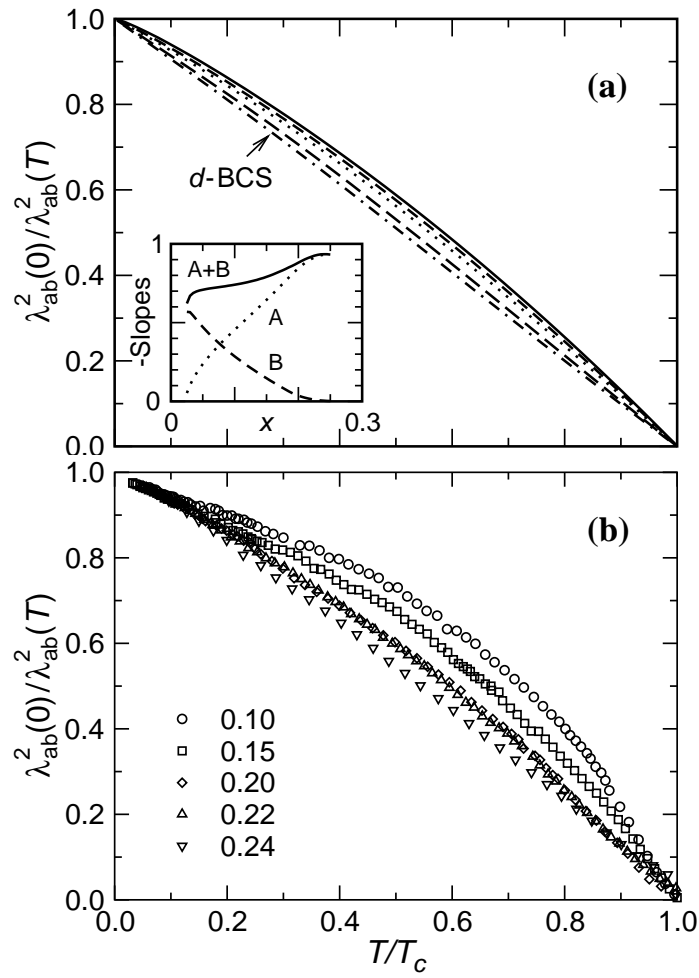

Figure 2. (a) Theoretical predictions for and (b) experimental measurements of the temperature dependence of the ab-plane inverse squared penetration depth. Systematic trends with hole concentration are similar. In (a), from bottom to top: $x=0.25$ (BCS limit, dot-dashed line), 0.2 (long-dashed), 0.155 (dotted), 0.125 (dashed) and 0.05 (solid line). At low $T, \lambda_{L}^{2}(0) / \lambda_{L}^{2}(T)=1-[A+$ $B(T)]\left(T / T_{c}\right)$, where $B(T) \propto \sqrt{T / T_{c}}$ depends on $T$ very weakly. Shown in the inset are the low temperature values for A and B. Experimental data on LSCO shown in (b) are taken from Ref. [19].

the question of what is responsible for the signature of true phase coherence at $T_{c}$, given that an excitation gap is present when superconductivity is established. Our recent work[10] has addressed this question in more quantitative detail. One can view the excitations of the normal state as consisting of fermions as well as bosons or pairs of fermions. The non-zero $Q$ (finite lifetime or "resonant") pair excitations persist below $T_{c}$ as well, and are not dramatically affected by the onset of phase coherence. However, the $Q=0$ pairs which Bose condense at $T_{c}$ necessarily acquire an infinite lifetime. It is this latter effect which is responsible for the signatures of coherence at $T_{c}$ in phase 
Page 4 of 4

insensitive measurements.

Clearly one of the most important questions to be addressed by the community is to determine whether the pseudogap phase derives from the superconductivity or from a competing order parameter. Evidence for the existence of a quantum critical point would seem consistent with the latter alternative, although it has been recently claimed[22] that thermodynamical data may not provide this support. In favor of the precursor scenario are the observations that the pseudogap has the same $d$-wave symmetry as the superconducting order parameter, and that there is no evidence of a phase transition at $T^{*}$. In addition, the fact that the high temperature superconductors seem to belong to a relatively large class of "exotic" superconductors as seen by the Uemura plot[11] suggests that one should focus on generic features of these materials in order to understand their superconductivity. Among the most distinctive generic features of the cuprates and other exotic superconductors is their short coherence length. It is this latter property which provides a very likely reason for the failure of BCS theory, and which underpins the present theoretical approach to the pseudogap phase.

We conjecture that the distinction between the two alternative origins of the pseudogap may be most clearly evident in the vicinity of the superconductorinsulator (SI) boundary. It is well established that the zero temperature excitation gap $\Delta(0)$ is large at this point, but the competing order parameter scenario[ [4] requires that the zero temperature order parameter $\Delta_{s c}(0)$ is vanishingly small. By contrast, in the absence of quantum fluctuations, the precursor scenario will have $\Delta_{s c}(0)=\Delta(0)$, which is thus large as well. In our rendition of this precursor school, superconductivity disappears because of the localization of $d$ wave pairs \&8. The fermionic excitation gap, which is much more robust, persists even after superconductivity is suppressed, so that the system becomes insulating once superconductivity is destroyed by doping, by magnetic fields or by $\mathrm{Zn}$ or other impurity substitutions. This ubiquitous SI transition should hold fundamental clues to the origin of the pseudogap and more generally to the non-BCS superconductivity of these materials.

This work was supported by the NSF-MRSEC, No. DMR-9808595 (KL, YK and AI), by the State of Florida (QC), by the University of Illinois (IK), and by University of Notre Dame (BJ).

\section{REFERENCES}

1. T. Timusk and B. Statt, Rep. Prog. Physics 62, 61 (1999).

2. J. L. Tallon and J. W. Loram, Physica C 349, 53 (2001).

3. Ch. Renner, B. Revaz, J.-Y. Genoud, K. Kadowaki, and Ø. Fischer, Phys. Rev. Lett. 80, 149 (1998).

4. S. Chakravarty, R. B. Laughlin, D. K. Morr, and C. Nayak, Phys. Rev. B 63, 094503 (2001).

5. B. Jankó, J. Maly, and K. Levin, Phys. Rev. B 56, R11 407 (1997); J. Maly, B. Jankó, and K. Levin, ibid. 59, 1354 (1999); Physica C 321, 113 (1999).

6. I. Kosztin, Q. J. Chen, B. Jankó, and K. Levin, Phys. Rev. B 58, R5936 (1998).

7. Q. J. Chen, I. Kosztin, B. Jankó, and K. Levin, Phys. Rev. Lett. 81, 4708 (1998).

8. Q. J. Chen, I. Kosztin, B. Jankó, and K. Levin, Phys. Rev. B 59, 7083 (1999).

9. Q. J. Chen, I. Kosztin, and K. Levin, Phys. Rev. Lett. 85, 2801 (2000).

10. Q. J. Chen, K. Levin, and I. Kosztin, Phys. Rev. B 63, 184519 (2001).

11. Y. J. Uemura, Physica C 282-287, 194 (1997).

12. A. J. Leggett, in Modern Trends in the Theory of Condensed Matter, edited by A. Pekalski and J. Przystawa (Springer-Verlag, Berlin, 1980), pp. 13-27.

13. P. Nozières and S. Schmitt-Rink, J. Low Temp. Phys. 59, 195 (1985).

14. M. Randeria, in Bose Einstein Condensation, ed. A. Griffin, D. Snoke, and S. Stringari (Cambridge University Press, Cambridge, UK, 1995), pp. 355-92.

15. V. J. Emery and S. A. Kivelson, Nature (London) 374, 434 (1995).

16. V. B. Geshkenbein, L. B. Ioffe, and A. I. Larkin, Phys. Rev. B 55, 3173 (1997).

17. L. P. Kadanoff and P. C. Martin, Phys. Rev. 124, 670 (1961).

18. B. R. Patton, Ph.D Thesis, Cornell University, 1971 (unpublished).

19. C. Panagopoulos et al., Phys. Rev. B 60, 14617 (1999).

20. B. R. Boyce, K. M. Paget, and T. R. Lemberger, condmat/9907196.

21. The magnetic field effects are discussed in a companion paper in this proceedings.

22. J. W. Loram, presentation at this conference (SNS 2001).

23. N. Miyakawa, Phys. Rev. Lett. 80, 157 (1998); 83, 1018 (1999).

24. J. Rossat-Mignod et al., Physica B 169, 58 (1991).

25. M. Oda et al., Physica C 281, 135(1997). 